\title{
Book-Oriented Environment in Childhood and Current Cognitive Performance among Old-Aged Europeans
}

\author{
Galit Weinstein $^{a} \quad$ Ella Cohn-Schwartz ${ }^{b}$ Noam Damri ${ }^{c}$ \\ aSchool of Public Health, Faculty of Social Welfare and Health Sciences, University of Haifa, Haifa, Israel; \\ ${ }^{b}$ Department of Public Health, Faculty of Health Sciences, Ben-Gurion University of the Negev, Beer-Sheva, Israel; \\ Israel Gerontological Data Center, Paul Baerwald School of Social Work and Social Welfare, The Hebrew University \\ of Jerusalem, Jerusalem, Israel
}

\section{Keywords}

Cognitive function · Early-life factors · Epidemiology • Longitudinal study

\begin{abstract}
Introduction: Identifying early-life factors that protect against compromised late-life cognition is of great public health interest. We aimed to explore the associations between book-oriented environment in childhood and late-life cognitive performance in the Survey of Health, Ageing and Retirement in Europe (SHARE). Methods: The sample included 8,239 individuals aged $\geq 65$ years $(N=8,239)$ free of stroke, Parkinson's disease, or Alzheimer's disease, who participated in both waves 4 (2011) and 5 (2013) of SHARE. Book-oriented environment was assessed by the self-reported home library size during childhood. Cognitive performance was assessed using tests of memory and verbal fluency. Covariates included education and measures of current health, lifestyle, and financial status. Additionally, interactions with age and education were assessed. Results: After controlling for potential confounders, having large home libraries was related to better performance on the immediate and delayed memory $(\beta=0.11 \pm 0.02, p<0.001 ; \beta=0.13 \pm 0.02, p<0.001)$ and
\end{abstract}

karger@karger.com www.karger.com/dem

Karger $\stackrel{\text { ' }}{5}$
(C) 2021 The Author(s)

Published by S. Karger AG, Basel

This is an Open Access article licensed under the Creative Common Attribution-NonCommercial-4.0 International License (CC BY-NC) (http://www.karger.com/Services/OpenAccessLicense), applicable to the online version of the article only. Usage and distribution for commercial purposes requires written permission. the verbal fluency tests $(\beta=0.14 \pm 0.06, p<0.001)$ and to a lesser decline in these domains $(\beta=0.08 \pm 0.01, p<0.001 ; \beta$ $=0.09 \pm 0.02, p<0.001$; and $\beta=0.09 \pm 0.06, p<0.001$, respectively). Significant interactions were observed between library size and age such that larger home library was more strongly associated with improved immediate memory ( $p=$ $0.016)$, delayed memory $(p<0.001)$, and verbal fluency $(p=$ $0.003)$ and with less cognitive decline $(p=0.013, p<0.001$, and $p=0.095$, respectively) among the younger-old $(<80$ years) compared to the oldest-old ( $\geq 80$ years) participants. No effect modification by education was observed. Conclusions: These findings suggest that early-life book-oriented environment may be important in shaping cognitive aging.

(c) 2021 The Author(s).

Published by S. Karger AG, Basel

\section{Introduction}

Cognitive decline is a prominent condition that can occur with aging [1] and may encompass various cognitive domains including processing speed, reasoning, memory, and executive function [2]. Life-time experiences, such as education attainment or engagement in social 
and occupational activities, have been linked with risk reduction of cognitive decline [3]. These factors are thought to increase cognitive reserve [4], which in turn enables a larger burden of brain pathologies before deficits begin to be apparent [5]. Although cognitive reserve is seen as a dynamic entity, which can be modified through cognitive stimulation throughout the life course, evidence suggests that the most influential period is during the first years of life, when key processes in brain development take place $[6,7]$. Indeed, indicators of early-life environment such as birth weight [8] and adulthood height $[9,10]$ have been linked with late-life cognitive performance. These morphometric measures reflect nutritional and health status during development in addition to genetic propensity [11]. Moreover, direct measures of early-life conditions such as childhood socioeconomic status, education, and perhaps most pronouncedly literacy $[12,13]$ are thought to delay the manifestation of cognitive impairment. Recent findings further suggest that cognitive enrichment in early life may be linked with better late-life cognitive health, which could partially be explained through changes in pathological processes in the brain [14].

Growing up with large home libraries reflects the parents' commitment to a book-oriented environment. Accumulating evidence suggests that home library size is positively associated with educational attainment [15], educational achievement $[16,17]$ and occupational success [18], which in turn have been linked with better cognitive function in late life [19]. A recent study among adults aged 25-65 years from 31 societies demonstrated a link between growing up with home libraries and improved cognitive skills (i.e., literacy, numeracy, and technological problem solving), independently of one's own or parental education or occupational attainment [20]. In the current study, we sought to assess the relationship between book-oriented environment as indicated by home library size in childhood and cognitive performance and decline in old individuals (aged $\geq 65$ years) who participated in the Survey of Health, Ageing and Retirement in Europe (SHARE) [21] and examined potential effect modification by age and education.

\section{Methods}

\section{Study Sample}

SHARE is a multidisciplinary cross-national, longitudinal survey which collects information on nationally representative samples of the community-dwelling population from 27 European countries and Israel. The target population of this survey was noninstitutionalized individuals aged 50 years and older and their spouses of any age. To date, data have been collected biennially during 6 waves, beginning in 2004 [22], using a computer-assisted personal interviewing program, supplemented by a self-completed paper-and-pencil questionnaire. The current analysis focused exclusively on participants aged 65 years and above who participated in the latest 2 waves of the survey for which data have been publicly released: wave 4 (2011) and wave 5 (2013). Wave 4 collected a wide range of data on health, socioeconomic status, and social and family networks from 16 European countries (Austria, Belgium, Czech Republic, Denmark, Estonia, France, Germany, Hungary, Italy, The Netherlands, Poland, Portugal, Slovenia, Spain, Sweden, and Switzerland) [23]. Wave 5 additionally included information on childhood conditions.

The current analytical sample was limited to age relevant participants who participated in both waves 4 and $5(N=19,314)$. We excluded 1,839 participants who reported being diagnosed with a stroke, Parkinson's disease, or Alzheimer's disease in wave 5, resulting in an initial sample of 17,475 . We then retained only individuals who answered the retrospective questions on childhood conditions. In wave 5 , this retrospective information was gathered only from participants who did not participate in SHARE prior to wave 4 , and thus our sample narrowed to 9,991 individuals. We further excluded 1,752 individuals who had missing information on the study's variables, and thus the main analytical sample for the current analysis included 8,239 participants. Informed consent was obtained from all participants prior to the interview [23].

\section{Measurements}

\section{Cognitive Performance}

The cognitive evaluation included 4 cognitive tests. Immediate and delayed episodic memory was assessed using the modified version of Rey's Auditory Verbal Learning Test - RAVLT [24], which evaluates short-term verbal learning and memory and information retention. In the modified version used in SHARE, the interviewer reads out a list of 10 words, after which the respondent is asked to recall as many of them as s/he can. The test is then repeated after 5-10 min. In the verbal fluency test, the participants were required to state the names of as many animals as they could, without repetitions or proper nouns, in a 1 -min period. Due to outliers in a small number of cases, scores above 45 were re-coded as 45 . This test measures executive functioning and language ability [25].

\section{Childhood Conditions}

Information on home library size in childhood was first collected in wave 5 of SHARE (2008/9), which focused on gathering retrospective information during the life course. This variable was assessed using the 5-scale item "How many books, approximately, were in your residence when you were 10 years or younger? (very few or none [ $0-10$ books], 1 shelf [11-25 books], 1 bookcase [26100 books], 2 bookcases [101-200 books], or $>2$ bookcases [200 books]).”

\section{Covariates}

Education level was based on participants' highest educational degree using the International Standard Classification of Education (ISCED-97) [26]. These classifications were then regrouped into 3 categories of low, medium, and high educational attainment as has been previously done [27]. Height and weight were assessed by self-report, and BMI was defined as weight (kg) 
divided by the square of height $(\mathrm{m})$. The number of chronic conditions was assessed by asking the respondents if a doctor ever told them that they had any of the conditions listed on a card, namely, cardiovascular disease, high blood pressure, high blood, cholesterol, stroke or cerebrovascular disease, diabetes, chronic lung disease, asthma, arthritis or rheumatism, osteoporosis, cancer or malignant tumor, stomach or duodenal or peptic ulcer, Parkinson disease, cataracts, and hip or femoral fracture. Participants could choose from 20 conditions or provide a different condition that was not listed in the questionnaire. Self-perceived health and hearing difficulties were assessed on a range from 1 to 5 , in which higher score indicated poorer status, and were included as covariates in the analyses. A participant was considered as physically inactive if he/she reported not doing moderate or vigorous physical activity at least once per week. Current financial status was assessed using 2 measures: (1) self-reported household wealth, which was imputed in SHARE, as the monetary variables were affected by nonnegligible amounts of missing data (http://share.cerge-ei.cz/documentation/Wave2004/SHARE_ w1_Imputation_of_Missing_Data_in_Waves_1_and_2_of_ SHARE.pdf). In addition, household wealth was adjusted for purchasing power parity and split into quintiles, calculating the quintile limits for each country separately. (2) Self-perceived financial status was measured by asking the participants about the extent to which they experienced difficulty in making ends meet. The scale ranged between 1 (difficult) and 4 (easy). This measure has been found to work effectively as a relative income indicator that can be compared across countries in which incomes have differing purchasing power [28]. In addition, respondents were asked whether they currently smoke cigarettes and whether or not they had a partner (spouse or registered partnership). Last, depressive symptoms (0-12) were measured using the Euro-D depression scale [29].

\section{Statistical Analyses}

Data analysis was conducted using STATA (version 13) [30]. Linear regression models were used to evaluate the associations of the number of books with cognitive performance on the immediate and delayed word list recall and the fluency tests. We also assessed the relationship of the number of books with change in cognitive performance between waves 4 and 5. For this purpose, we subtracted the test score in wave 5 from the score in wave 4 and ran linear regression models with the change as the outcome, while controlling for the variable at baseline. In addition, we tested whether current age and education modify the associations between number of books and cognitive function by including interaction terms for these variables in the linear regression model. If a significant interaction was found, we ran a stratified model. $p$ value cutoff for interaction was set to be 0.10 due to low power of statistical interaction tests. For age stratification, we chose the cutoff of 80 years. Models were adjusted first for age, sex, education, and country. Subsequent models were additionally adjusted for BMI, number of chronic conditions, perceived health, perceived financial status, household wealth, hearing difficulties, having a partner, physical inactivity, and smoking status. Of note, although financial status in childhood was considered a potential confounder, we did not adjust for it because it was not associated with cognitive function and decline in our sample and was highly correlated with other covariates (data not shown).
Table 1. Characteristics of study population (wave 5)

\begin{tabular}{ll} 
Subjects, $N$ & 8,239 \\
Age, years & $73 \pm 6.3$ \\
Women & $4,531(55)$ \\
Education & \\
$\quad$ Less than high school & $2,966(36)$ \\
$\quad$ High school & $2,966(36)$ \\
$\quad$ Higher education & $2,307(28)$ \\
Has a partner & $5,190(63)$ \\
BMI & $27 \pm 4.4$ \\
Chronic conditions (0-20), $n$ & $1.9 \pm 1.5$ \\
Perceived health (1-5) & $2.7 \pm 1.0$ \\
Physical inactivity & $906(11)$ \\
Current smoking & $1,071(13)$ \\
Perceived financial adequacy (1-4) & $2.9 \pm 0.9$ \\
Quintiles of current household wealth, Euros & \\
$\quad$ 1 quintile (lowest) & 11,586 \\
$\quad 2$ quintile & 77,615 \\
$\quad 3$ quintile & 161,016 \\
$\quad 4$ quintile & 272,947 \\
$\quad 5$ quintile (highest) & 749,242 \\
Hearing difficulties (1-5) & $2.8 \pm 1.0$ \\
Depression (Euro-D; 0-12) & $2.3 \pm 2.1$ \\
Word list recall, immediate (0-10) & $5.3 \pm 1.7$ \\
Word list recall, delayed (0-10) & $3.8 \pm 2.1$ \\
Verbal fluency (0-45) & $20.7 \pm 6.8$ \\
Serial sevens test (\% all correct answers) & $5,685(69)$ \\
Books in childhood (1-5), $n$ & $2.2 \pm 1.2$ \\
\hline
\end{tabular}

Values are $n(\%)$ or mean \pm SD.

\section{Results}

The characteristics of the study participants in wave 5 are described in Table 1. Mean age of the responders was $73.0 \pm 6.3$ years and 4,531 (55\%) were women. Most participants reported having books that were "Enough to fill one shelf (11-25 books)" in their childhood.

Table 2 demonstrates the associations of library size in childhood with cognitive performance and rate of cognitive decline in late life. A larger library in childhood was related to better performance on the immediate and delayed memory test $(\beta=0.12 \pm 0.02, p<0.001$; and $\beta=0.14$ $\pm 0.02, p<0.001$, respectively), as well as on the fluency test $(\beta=0.15 \pm 0.06, p<0.001)$ in wave 5 independently of age, sex, education, and country of residence. These associations remained similar after additional adjustment for health, lifestyle, and financial factors $(\beta=0.11 \pm 0.02$, $p<0.001 ; \beta=0.13 \pm 0.02, p<0.001 ;$ and $\beta=0.14 \pm 0.06$ for immediate memory, delayed memory, and fluency, respectively). On average, change in cognitive performance between waves 4 and 5 was $0.17,0.06$, and -0.08 
Table 2. Cross-sectional and longitudinal associations between childhood library size and cognitive function $(N$ $=8,239)$

\begin{tabular}{|c|c|c|c|c|c|c|}
\hline & \multicolumn{2}{|c|}{ Memory - immediate recall } & \multicolumn{2}{|c|}{ Memory - delayed recall } & \multicolumn{2}{|l|}{ Fluency } \\
\hline & $\beta \pm \mathrm{SE}$ & $p$ value & $\beta \pm S E$ & $p$ value & $\beta \pm S E$ & $p$ value \\
\hline \multicolumn{7}{|c|}{ Cross-sectional in wave 5} \\
\hline Model 1 & $0.12 \pm 0.02$ & $<0.001$ & $0.14 \pm 0.02$ & $<0.001$ & $0.15 \pm 0.06$ & $<0.001$ \\
\hline Model 2 & $0.11 \pm 0.02$ & $<0.001$ & $0.13 \pm 0.02$ & $<0.001$ & $0.14 \pm 0.06$ & $<0.001$ \\
\hline \multicolumn{7}{|c|}{ Change between waves 4 and 5} \\
\hline Model 1* & $0.08 \pm 0.02$ & $<0.001$ & $0.09 \pm 0.02$ & $<0.001$ & $0.10 \pm 0.06$ & $<0.001$ \\
\hline Model 2 & $0.08 \pm 0.01$ & $<0.001$ & $0.09 \pm 0.02$ & $<0.001$ & $0.09 \pm 0.06$ & $<0.001$ \\
\hline
\end{tabular}

Model 1: adjusted for age, sex, education, and country. Model 2: model $1+$ number of chronic conditions, perceived health, perceived financial status, household wealth, hearing difficulties, partner, physical inactivity, smoking status, and depression. Values in bold indicate $p$ value $\leq 0.05 . *$ Additionally adjusted for cognitive score at baseline.

on the immediate, delayed recall, and fluency tests, respectively (not statistically significant). A large library at home during childhood was associated with slower cognitive decline in immediate recall, delayed recall, and fluency $(\beta=0.08 \pm 0.02, p<0.001 ; \beta=0.09 \pm 0.02, p<0.001$; and $\beta=0.10 \pm 0.06, p<0.001$, respectively), after adjusting for age, sex, education, and country of residence. Similar trends were observed in models adjusting for additional health, lifestyle, and financial factors $(\beta=0.08 \pm$ $0.01, p<0.001 ; \beta=0.09 \pm 0.02, p<0.001$; and $\beta=0.09 \pm$ $0.06, p<0.001$, respectively; Table 2 ).

We found statistically significant interactions between library size and current age in relation to cognitive performance in immediate recall ( $p$ for interaction $=0.016$ ), delayed recall ( $p$ for interaction $<0.001$ ), and fluency test ( $p$ for interaction $=0.003$ ) in wave 5. Similar interactions were found between age and number of books in relation to changes in immediate recall ( $p$ for interaction $=0.013$ ), delayed recall ( $p$ for interaction $<0.001$ ), and fluency, although it was borderline for the latter $(p$ for interaction $=$ 0.095) (Table 3; Fig. 1). For both the cross-sectional and longitudinal associations between library size and cognition, a stratified analysis revealed that the associations were stronger among those younger than 80 years of age (Table 3$)$. At the current age below 80 years, a larger home library was associated with an increase of $0.11 \pm 0.02(p<$ $0.001), 0.13 \pm 0.02(p<0.001)$, and $0.14 \pm 0.07(p<0.001)$ in immediate and delayed memory and in fluency, respectively, and with a slower cognitive decline in these domains $(0.09 \pm 0.02, p<0.001 ; 0.09 \pm 0.02, p<0.001$; and $0.10 \pm 0.06, p<0.001$, respectively) after adjustment for health, lifestyle, and financial factors (Table 3; Fig. 1).
No significant interactions were observed between library size and education in relation for cognitive function both cross-sectionally and longitudinally. While $p$ for interaction suggested a possible modifying effect for immediate memory performance and change in it ( $p$ for interaction 0.092 and 0.037 , respectively, comparing higher education to elementary and 0.012 and 0.006 comparing high school to elementary), stratified analyses revealed no meaningful differences in effect sizes between levels of education (Tables 2, 3).

\section{Discussion}

Our findings stress the possible contribution of home libraries to improved cognitive health and to slower brain aging decades later. Prior research shows that growing up in homes with larger libraries has benefits beyond that of education attainment. It is thought that children who are encouraged to read and enjoy books acquire cognitive skills that help them succeed [31]. Indeed, a recent study showed that exposure to books in early life fosters long-term cognitive competencies spanning literacy, numeracy, and technological problem solving at adulthood [20]. Thus, the association between library size and cognitive performance in old age shown in our study may be mediated through acquisition of cognitive skills. Literacy, in particular, has been related to latelife cognitive function as well as with neuropathological measures of brain aging in the Nun study [32]. Because linguistic abilities appeared to moderate the relationship between neuropathology and late-life cognition (i.e., for 
Table 3. Cross-sectional and longitudinal associations between childhood library size and cognitive function by age and level of education

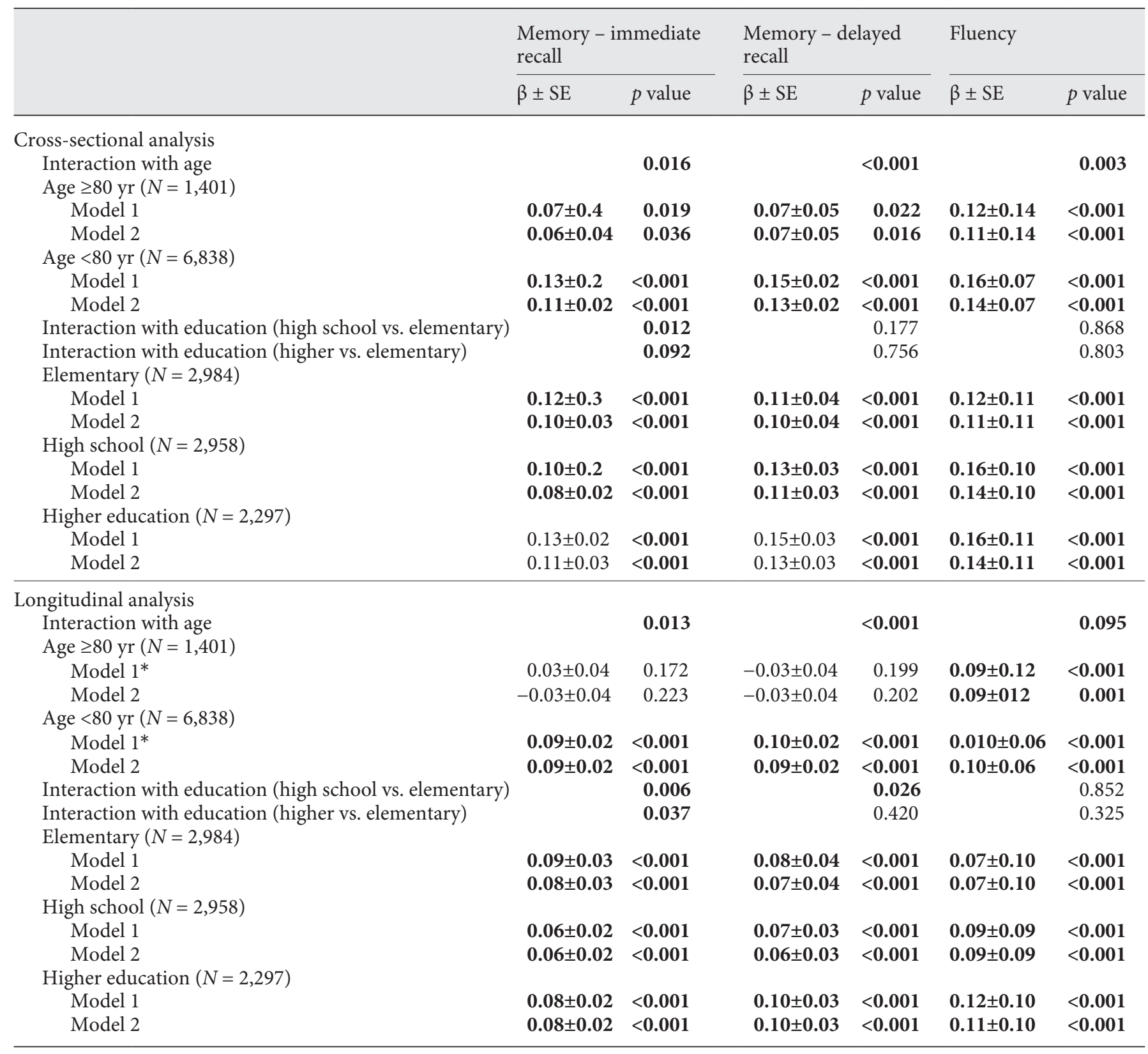

Model 1: adjusted for age, sex, education, and country. Model 2: model 1 + BMI, number of chronic conditions, perceived health, perceived financial status, household wealth, hearing difficulties, partner, physical inactivity, smoking status, and depression. Values in bold indicate $p$ value $\leq 0.05$. Additionally adjusted for cognitive score at baseline.

those with high linguistic capabilities, the association between brain pathology and cognitive status proximal to death is weaker), it is thought that early-life linguistic ability may act as a marker for cognitive reserve [33]. More recently, poor linguistic capabilities at young adulthood were associated with greater subsequent decline in global cognition, semantic memory, episodic memory, and spatial abilities in old age [34]. Thus, availability of books in early life may be an extrinsic factor affecting linguistic abilities [35] which in turn builds-up cognitive reserve [36] and results in improved cognition and slower cognitive decline. 


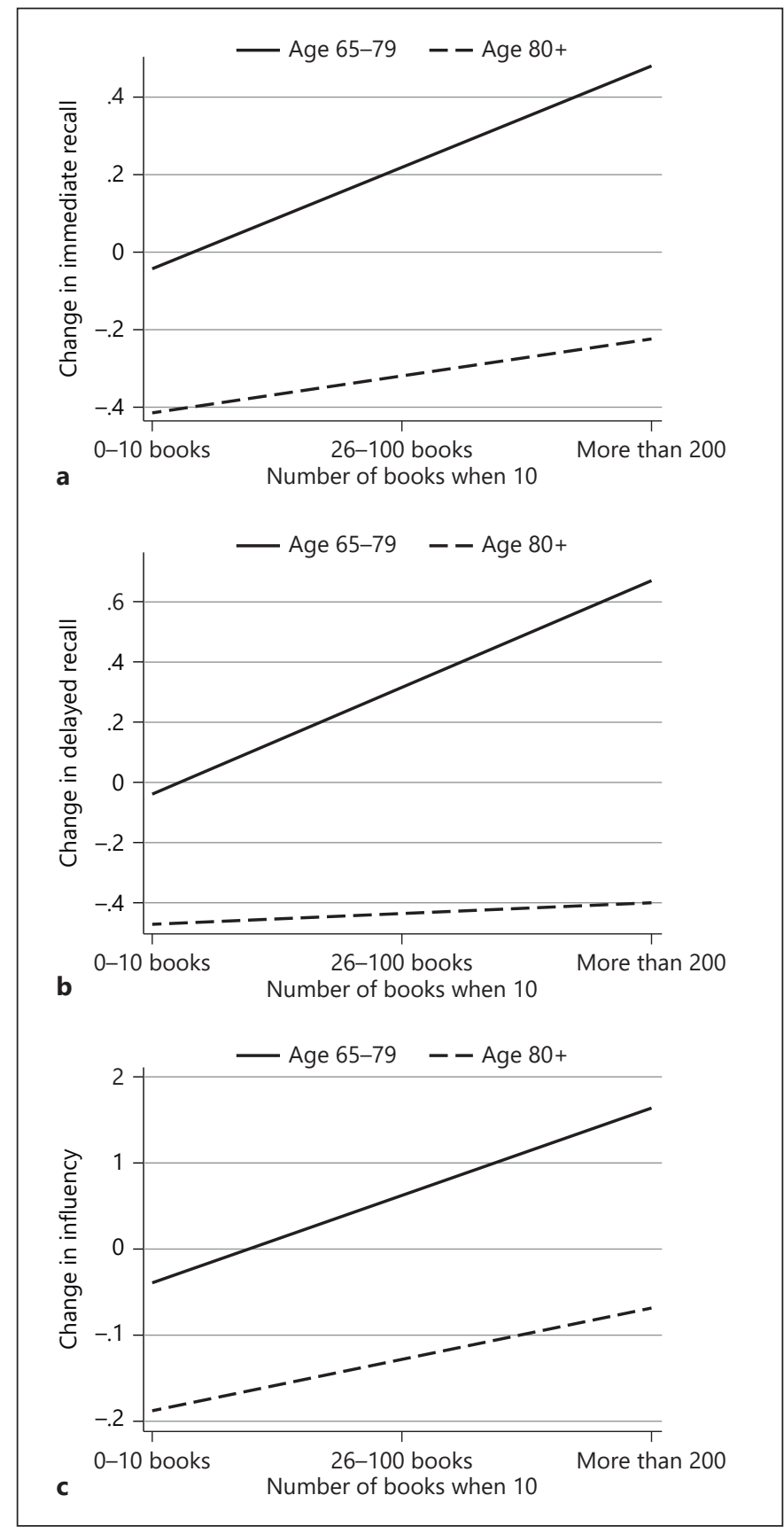

Fig. 1. The association between number of books and change in immediate memory (a), delayed memory (b), and fluency (c) by age at baseline (65-79 vs. $\geq 80$ years).

While the association between book-oriented environment and cognition in our study was independent of education per se, it is still possible that those who were exposed to many books at home maintained higher education [37], which resulted in greater mental activity in occupation and leisure pursuits throughout life [38]. In turn, accumulating evidence suggests that participation in cognitively stimulating activities protects against cognitive impairment and slows cognitive decline [39]. Support for this hypothesis also comes from findings from SHARE, showing that elderly individuals who are engaged in cognitively stimulating leisure activities such as crossword and Sudoku demonstrate better cognitive performance on the same cognitive tests assessed in the present study [40]. Even irrespective of education attainment, larger home libraries lay a foundation for life-long routine activities that enhance literacy and numeracy [20]. Furthermore, children who grew up with large home libraries perform better at school [31, 41] and work [18]. In turn, high childhood school performance is related to lower dementia risk in late life [42], and enriched environment at work has been associated with improved cognitive function in middle and old ages [43] as well as with slower rate of cognitive decline in old adults [44].

Exposure to a large number of books at home in early life may also influence health behaviors and lifestyle choices, which could subsequently result in late-life cognitive performance $[12,45-47]$. However, the fact that the link between library size and cognitive function and rate of decline remained similar after adjustment for health and lifestyle factors, together with the association with cognitive change between 2 measurements, implies that early-life exposure to books may be directly associated with brain aging. Further supports for this hypothesis arrive from recent findings showing that part of the association between early-life enrichment activities and cognitive aging is explained by less pathological changes in the brain [14].

Library size may be, at least partly, influenced by genetic variants such as those affecting education attainment $[48,49]$. According to large genome-wide association studies, some of these variants overlap with those for cognitive performance and are highly heritable [50]. Thus, our findings can also be explained by genetic propensity affecting both the exposure and outcome of our study through transmission of traits to the offspring generation.

We found that age modifies the association between number of books at home and cognitive performance as well as change in cognition over time, such that stronger associations were apparent in those younger than 80 years. These results may represent cohort effects, as clear trends exist with regard to time spent with leisure reading, and there is a shift toward using other media [51]. Alternatively, cognitive function among the oldest-old is 
largely influenced by life-course environmental, lifestyle, and health factors and less by early-life factors. No evidence of effect modification by education level was found in our study, which stands in contrast to others who have shown that early-life leisure time activity has greater benefits to the less educated [40].

The strengths of the current study include its longitudinal design with a relatively large and ethnically diverse sample. In addition, we were able to adjust for multiple potential confounders. The study also has several limitations: first, childhood conditions were self-reported, and thus misclassification within categories of library size may have occurred. Nevertheless, because all responders were free of dementia, such misclassification is likely nondifferential (unrelated to cognitive ability) and thereMoreover, self-report of childhood living conditions has been shown to be valid [52-54]. Second, the study lacks information on brain imaging, which could add support to our conclusions. Last, residual confounding and possible interactions between covariates could affect our results.

\section{Conclusion}

There is a growing understanding that cognitive health at old ages begins at conception [55]. Thus, identifying early-life factors that influence cognitive function among the growing elderly population is of great importance. Our study shows that availability of books at home during childhood may be related to improved late-life cognitive abilities and to slower cognitive decline, independently of education and life-course factors such as health, lifestyle, and socioeconomic indices. Future research involving neuroimaging is warranted to clarify the possible underlying mechanisms. In addition, the transition from printed to digital material as a proxy for home book-oriented environment should be taken into account.

\section{Statement of Ethics}

The SHARE study is subject to continuous ethics review. Wave 4 and the continuation of the project were reviewed and approved by the Ethics Council of the Max Planck Society. In addition, the country implementations of SHARE were reviewed and approved by the respective ethics committees or institutional review boards whenever this was required. The numerous reviews covered all aspects of the SHARE study, including subprojects, and confirmed the project to be compliant with the relevant legal norms and that the project and its procedures agree with international ethical stanfore results in an underestimation of the real association.

dards. Informed consent was obtained from all participants. Overview and summary of the ethics approvals can be found in the following link: http://www.share-project.org/fileadmin/pdf_documentation/SHARE_ethics_approvals.pdf.

\section{Conflict of Interest Statement}

The authors have no conflicts of interest to declare.

\section{Funding Sources}

The SHARE data collection has been funded by the European Commission through the 5th framework programme (project QLK6-CT-2001-00360 in the thematic programme Quality of Life). Further support by the European Commission through the 6th framework programme (projects SHARE-I3, RIICT-2006-062193, as an Integrated Infrastructure Initiative, COMPARE, CIT5-CT-2005-028857, as a project in Priority 7, Citizens and Governance in a Knowledge Based Society, and SHARE-LIFE [CIT4-CT-2006-028812]), through the 7th framework programme (SHARE-PREP [No. 211909], SHARE-LEAP [No. 227822], and M4 [No. 261982]), and through Horizon 2020 (SHAREDEV3 [No. 676536] and SERISS [No. 654221]) is gratefully acknowledged.

\section{Author Contributions}

G.W., E.C.-S., and N.D. were responsible for the study concept and design. E.C.-S. and N.D. analyzed the data. G.W. and E.C.-S. drafted the manuscript.

\section{Data Availability Statement}

Our study is a secondary analysis of existing data from the Survey of Health, Ageing and Retirement in Europe (SHARE). Data files and documentation are for public use and available at http:// www.share-project.org.
References
1 Petersen RC. Mild cognitive impairment as a diagnostic entity. J Intern Med. 2004;256(3): 183-94.

2 Deary IJ, Corley J, Gow AJ, Harris SE, Houlihan LM, Marioni RE, et al. Age-associated cognitive decline. Br Med Bull. 2009;92(1): $135-52$

3 Foubert-Samier A, Catheline G, Amieva H, Dilharreguy B, Helmer C, Allard M, et al. Education, occupation, leisure activities, and brain reserve: a population-based study. Neurobiol Aging. 2012;33(2):423-5.

4 Valenzuela MJ, Sachdev P. Brain reserve and cognitive decline: a non-parametric systematic review. Psychol Med. 2006;36(8):106573. 
5 Stern Y. Cognitive reserve in ageing and Alzheimer's disease. Lancet Neurol. 2012; 11(11):1006-12.

6 Fritsch T, McClendon MJ, Smyth KA, Lerner AJ, Friedland RP, Larsen JD. Cognitive functioning in healthy aging: the role of reserve and lifestyle factors early in life. Gerontologist. 2007;47(3):307-22.

7 Fox SE, Levitt P, Nelson CA 3rd. How the timing and quality of early experiences influence the development of brain architecture. Child Dev. 2010;81(1):28-40.

8 Strauss RS. Adult functional outcome of those born small for gestational age: twenty-six-year follow-up of the 1970 British Birth Cohort. JAMA. 2000;283(5):625-32.

9 Quan SA, Jeong JY, Kim DH. The relationship between height and cognitive function among community-dwelling elderly: Hallym Aging Study. Epidemiol Health. 2013; 35:e2013002.

10 West RK, Ravona-Springer R, Heymann A, Schmeidler J, Leroith D, Koifman K, et al. Shorter adult height is associated with poorer cognitive performance in elderly men with type II diabetes. J Alzheimers Dis. 2015;44(3):927-35.

11 Bogin B, Varela-Silva MI. Leg length, body proportion, and health: a review with a note on beauty. Int J Environ Res Public Health. 2010;7(3):1047-75.

12 Jefferson AL, Gibbons LE, Rentz DM, Carvalho JO, Manly J, Bennett DA, et al. A life course model of cognitive activities, socioeconomic status, education, reading ability, and cognition. J Am Geriatr Soc. 2011; 59(8):1403-11.

13 Manly JJ, Touradji P, Tang MX, Stern Y. Literacy and memory decline among ethnically diverse elders. J Clin Exp Neuropsychol. 2003;25(5):680-90.

14 Oveisgharan S, Wilson RS, Yu L, Schneider JA, Bennett DA. Association of early-life cognitive enrichment with alzheimer disease pathological changes and cognitive decline. JAMA Neurol. 2020;77(10):1217-24.

15 Evans M, Kelley J, Sikora J, Treiman DJ. Scholarly culture and educational success in 27 nations. Canberra: Australian National University; 2005.

16 Evans MDR, Kelley J, Sikora J. Scholarly culture and academic performance in 42 nations. Social Forces. 2014;92(4):1573605.

17 Manu A, Ewerling F, Barros AJ, Victora CG. Association between availability of children's book and the literacy-numeracy skills of children aged 36 to 59 months: secondary analysis of the UNICEF MultipleIndicator Cluster Surveys covering 35 countries. J Glob Health. 2019;9(1):010403.

18 Evans MDR, Kelley J, Sikora J, Treiman DJ. Scholarly culture and occupational success in 31 societies. Comp Sociol. 2015;14(2): 176-218.
19 Fujishiro K, MacDonald LA, Crowe M, McClure LA, Howard VJ, Wadley VG. The role of occupation in explaining cognitive functioning in later life: education and occupational complexity in a US national sample of Black and White Men and Women. J Gerontol B Psychol Sci Soc Sci. 2019;74(7): 1189-99.

20 Sikora J, Evans MDR, Kelley J. Scholarly culture: how books in adolescence enhance adult literacy, numeracy and technology skills in 31 societies. Soc Sci Res. 2019;77: 1-15.

21 Börsch-Supan A, Brandt M, Hunkler C, Kneip T, Korbmacher J, Malter F, et al. Data resource profile: the survey of health, ageing and retirement in Europe (SHARE). Int J Epidemiol. 2013;42(4):992-1001.

22 Börsch-Supan A, Brandt M, Hunkler C, Kneip T, Korbmacher J, Malter F, et al. Data resource profile: the survey of health, ageing and retirement in Europe (SHARE). Int J Epidemiol. 2013 Aug;42(4):992-1001.

23 Abduladze L, Malter F, Börsch-Supan A. SHARE wave 4: innovations \& methodology. Munich, Germany: Munich Center for the Economics of Aging; 2013.

24 Schoenberg MR, Dawson KA, Duff K, Patton D, Scott JG, Adams RL. Test performance and classification statistics for the Rey Auditory Verbal Learning Test in selected clinical samples. Arch Clin Neuropsychol. 2006;21(7):693-703.

25 Tombaugh TN, Kozak J, Rees L. Normative data stratified by age and education for two measures of verbal fluency: FAS and animal naming. Arch Clin Neuropsychol. 1999; 14(2):167-77.

26 UNESCO. International standard classification of education : ISCED 1997. Paris: UNESCO; 1997. p. 42

27 Lunau T, Siegrist J, Dragano N, Wahrendorf $\mathrm{M}$. The association between education and work stress: does the policy context matter? PLoS One. 2015;10(3):e0121573.

28 Litwin H, Sapir EV. Perceived income adequacy among older adults in 12 countries: findings from the survey of health, ageing, and retirement in Europe. Gerontologist. 2009;49(3):397-406

29 Prince MJ, Reischies F, Beekman AT, Fuhrer R, Jonker C, Kivela SL, et al. Development of the EURO-D scale--a European, Union initiative to compare symptoms of depression in 14 European centres. Br J Psychiatry. 1999;174:330-8.

30 Stata Corp. Stata statistical software: release 13; 2013.

31 Evans MDR, Kelley J, Sikora J. Scholarly culture and academic performance in 42 nations. Social Forces. 2014;92(4):1573605.

32 Riley KP, Snowdon DA, Desrosiers MF, Markesbery WR. Early life linguistic ability, late life cognitive function, and neuropathology: findings from the Nun Study. Neurobiol Aging. 2005;26(3):341-7.
33 Iacono D, Markesbery WR, Gross M, Pletnikova O, Rudow G, Zandi P, et al. The Nun study: clinically silent $\mathrm{AD}$, neuronal hypertrophy, and linguistic skills in early life. Neurology. 2009;73(9):665-73

34 Farias ST, Chand V, Bonnici L, Baynes K, Harvey D, Mungas D, et al. Idea density measured in late life predicts subsequent cognitive trajectories: implications for the measurement of cognitive reserve. J Gerontol B Psychol Sci Soc Sci. 2012;67(6):67786.

35 Dickinson DK, Griffith JA, Golinkoff RM, Hirsh-Pasek K. How reading books fosters language development around the world. Child Development Research. 2012;2012:1.

36 Stern Y, Barulli D. Cognitive reserve. In: Dekosky ST, Asthana S, editors. Handbook of clinical neurology. Elsevier; 2019. Vol. 167; p. 181-90.

37 Evans MDR, Kelley J, Sikora J, Treiman DJ. Family scholarly culture and educational success: books and schooling in 27 nationsResearch in social stratification and mobility. Res Soc Stratif Mobil. 2010;28(2):17197.

38 Kramer AF, Bherer L, Colcombe SJ, Dong W, Greenough WT. Environmental influences on cognitive and brain plasticity during aging. J Gerontol A Biol Sci Med Sci. 2004;59(9):M940-57.

39 Cheng ST. Cognitive reserve and the prevention of dementia: the role of physical and cognitive activities. Curr Psychiatry Rep. 2016;18(9):85.

40 Litwin H, Schwartz E, Damri N. Cognitively stimulating leisure activity and subsequent cognitive function: a SHARE-based analysis. Gerontologist. 2017;57(5):940-8.

41 Park H. Home literacy environments and children's reading performance: a comparative study of 25 countries. Educ Res Eval. 2008;14(6):489-505.

42 Dekhtyar S, Wang HX, Fratiglioni L, Herlitz A. Childhood school performance, education and occupational complexity: a lifecourse study of dementia in the Kungsholmen Project. Int J Epidemiol. 2016;45(4): 1207-15.

43 Then FS, Luck T, Luppa M, Arélin K, Schroeter ML, Engel C, et al. Association between mental demands at work and cognitive functioning in the general population: results of the health study of the Leipzig research center for civilization diseases (LIFE). J Occup Med Toxicol. 2014;9: 23.

44 Then FS, Luck T, Luppa M, König HH, Angermeyer MC, Riedel-Heller SG. Differential effects of enriched environment at work on cognitive decline in old age. Neurology. 2015;84(21):2169-76. 
45 Horvat P, Richards M, Malyutina S, Pajak A, Kubinova R, Tamosiunas A, et al. Life course socioeconomic position and midlate life cognitive function in Eastern Europe. J Gerontol B Psychol Sci Soc Sci. 2014; 69(3):470-81.

46 Luo Y, Waite LJ. The impact of childhood and adult SES on physical, mental, and cognitive well-being in later life. J Gerontol B Psychol Sci Soc Sci. 2005;60(2):S93-101.

47 Mirowsky J, Ross CE. Education, social status, and health. New York: A. de Gruyter; 2003. p. vii, 242.

48 Okbay A, Beauchamp JP, Fontana MA, Lee JJ, Pers TH, Rietveld CA, et al. Genomewide association study identifies 74 loci associated with educational attainment. Nature. 2016;533(7604):539-42.
49 Rietveld CA, Medland SE, Derringer J, Yang J, Esko T, Martin NW, et al. GWAS of 126,559 individuals identifies genetic variants associated with educational attainment. Science. 2013;340(6139):1467-71.

50 Trampush JW, Yang ML, Yu J, Knowles E, Davies G, Liewald DC, et al. GWAS metaanalysis reveals novel loci and genetic correlates for general cognitive function: a report from the COGENT consortium. Mol Psychiatry. 2017;22(3):336-45.

51 Knulst W, Kraaykamp G. The decline of reading. Leisure reading trends in the Netherlands (1955-1995). Neth J Soc Sci. 1997; 33:130-50.
52 Batty GD, Lawlor DA, Macintyre S, Clark $\mathrm{H}$, Leon DA. Accuracy of adults' recall of childhood social class: findings from the Aberdeen children of the 1950s study. J Epidemiol Community Health. 2005;59(10): 898-903.

53 Berney LR, Blane DB. Collecting retrospective data: accuracy of recall after 50 years judged against historical records. Soc Sci Med. 1997;45(10):1519-25.

54 Havari E, Mazzonna F. Can we trust older people's statements on their childhood circumstances? Evidence from SHARELIFE. Eur J Popul. 2015;31(3):233-57.

55 Barnett JH, Hachinski V, Blackwell AD. Cognitive health begins at conception: addressing dementia as a lifelong and preventable condition. BMC Med. 2013;11:246. 